\title{
INEQUALITIES IN SUMMABILITY THEORY OF FOURIER SERIES
}

\section{FERENC WEISZ}

Abstract. Some recent results on a general summability method, the so-called $\theta$-summability, are summarized for one-dimensional Fourier series. Natural choices of $\theta$ are investigated, i.e., if $\theta$ is in Wiener amalgam spaces, Feichtinger's algebra or modulation spaces. Sufficient and necessary conditions are given for the uniform and $L_{1}$ norm and a.e. convergence of the $\theta$ means $\sigma_{n}^{\theta} f$ to the function $f$. The maximal operator of the $\theta$-means is investigated and it is proved that it is bounded on $L_{p}$ spaces and on Hardy spaces.

Mathematics subject classification (2000): Primary 42B08, 46E30, Secondary 42B30, 42A38.

Keywords and phrases: Wiener amalgam spaces, Feichtinger's algebra, modulation spaces, Herz and Hardy spaces, $\theta$-summability, Lebesgue points.

\section{REFERENCES}

[1] J. Bergh AND J. LÖfSTRÖm, Interpolation Spaces, an Introduction, Springer, Berlin, 1976.

[2] P. L. Butzer And R. J. Nessel, Fourier Analysis and Approximation, Birkhäuser Verlag, Basel, 1971.

[3] L. CARLESON, On convergence and growth of partial sums of Fourier series, Acta Math., 116 (1966), $135-157$.

[4] H. G. Feichtinger And F. Weisz, The Segal algebra $\mathbf{S}_{0}\left(\mathbb{R}^{d}\right)$ and norm summability of Fourier series and Fourier transforms, Monatshefte Math., 148 (2006), 333-349.

[5] H. G. Feichtinger And F. Weisz, Wiener amalgams and pointwise summability of Fourier transforms and Fourier series, Math. Proc. Camb. Phil. Soc., 140 (2006), 509-536.

[6] H. G. Feichtinger And G. Zimmermann, A Banach space of test functions for Gabor analysis, In H. G. Feichtinger and T. Strohmer, editors, Gabor Analysis and Algorithms. Theory and Applications, Applied and Numerical Harmonic Analysis, pp. 123-170, Boston, MA, 1998. Birkhäuser. chap. 3.

[7] L. FEJÉR, Untersuchungen über Fouriersche Reihen, Math. Annalen, 58 (1904), 51-69.

[8] K. GRÖCHEnIG, Foundations of Time-Frequency Analysis, Birkhäuser, Boston, 2001.

[9] Y. Katznelson, An Introduction to Harmonic Analysis, Cambridge Mathematical Library. Cambridge University Press, 3rd edition, 2004.

[10] H. LeBESGUE, Recherches sur la convergence des séries de Fourier, Math. Annalen, 61 (1905), 251280.

[11] J. MarcinkiewicZ And A. Zygmund, On the summability of double Fourier series, Fund. Math., 32 (1939), 122-132.

[12] G. M. NAtanson And V. V. Zuk, Trigonometric Fourier Series and Elements of Approximation Theory, Leningrad. Univ., Leningrad, 1983. (in Russian)

[13] E. M. SteIn, Harmonic Analysis: Real-variable Methods, Orthogonality and Oscillatory Integrals, Princeton Univ. Press, Princeton, N.J., 1993.

[14] R. M. Trigub And E. S. Belinsky, Fourier Analysis and Approximation of Functions, Kluwer Academic Publishers, Dordrecht, Boston, London, 2004.

[15] F. WeIsZ, $\theta$-summation and Hardy spaces, J. Appr. Theory, 107 (2000), 121-142.

[16] F. WeIsz, Summability of Multi-dimensional Fourier Series and Hardy Spaces, Mathematics and Its Applications, Kluwer Academic Publishers, Dordrecht, Boston, London, 2002.

[17] F. WeIsZ, Wiener amalgams, Hardy spaces and summability of Fourier series, Math. Proc. Camb. Phil. Soc., 145 (2008), 419-442. 